\title{
A Comprehensive Atomistic Analysis of Bandstructure Velocities in Si Nanowires
}

\author{
Neophytos Neophytou and Hans Kosina \\ Institute for Microelectronics, \\ Technical University of Vienna, TU Wien \\ Vienna, Austria \\ \{neophytou|kosina\}@iue.tuwien.ac.at
}

\author{
Gerhard Klimeck \\ Network for Computational Nanotechnology \\ Purdue University \\ West Lafayette, IN, USA \\ gecko@purdue.edu
}

\begin{abstract}
A 20 band $\mathbf{s p}^{3} \mathbf{d}^{5} \mathbf{s}^{*}$ spin-orbit-coupled, semi-empirical, atomistic tight-binding (TB) model is used with a semi-classical, ballistic transport model, to theoretically examine the bandstructure carrier velocity under non-degenerate conditions in silicon nanowire (NW) transistors. Infinitely long, uniform, cylindrical and rectangular NWs, of cross sectional diameters/sides ranging from $3 \mathrm{~nm}$ to $12 \mathrm{~nm}$ are considered. For a comprehensive analysis, n-type and p-type NWs in [100], [110] and [111] transport orientations are examined. The carrier velocities of p-type [110] and [111] NWs increase by a factor of $\sim 2 X$ as the NWs' diameter scales from $D=12 \mathrm{~nm}$ down to $D=3 \mathrm{~nm}$. The velocity of n-type [110] NWs also increases with diameter scaling by $\sim 50 \%$. The velocities of n-type [100], and [111], as well as those of p-type [100] NWs show only minor diameter dependence. This behavior is explained through features in the electronic structure of the silicon host material.
\end{abstract}

Keywords- nanowire, velocity, atomistic, bandstructure, $s p^{3} d^{5} s^{*}$, tight binding, transistors, MOSFETs, variations, effective mass.

\section{INTRODUCTION}

Silicon nanowires (NWs) are candidates for a variety of applications such as high performance MOSFET [1, 2, 3], thermoelectric [4, 5], optical [6] and biosensing devices [7]. NWs provide the length scale degree of freedom as an additional design parameter for their electronic properties. The carrier velocity is an important quantity for both, short-channel ballistic, or long-channel diffusive devices by affecting the current or the mobility, respectively.

In this work we present a comprehensive atomistic analysis of the bandstructure velocities in thin NWs for electrons and holes as a function of diameter (from $D=3 \mathrm{~nm}$ to $D=12 \mathrm{~nm}$ ) and orientation (transport/confinement). We also consider rectangular NWs of different side length aspect ratios. The $\mathrm{sp}^{3} \mathrm{~d}^{5} \mathrm{~s}$-spin-orbit-coupled atomistic tight-binding model is used to compute the electronic structure of the NWs $[8,9,10]$. A semiclassical ballistic model is used for the velocity calculation $[10,11]$.

The carrier velocities are a function of the electronic dispersion of the NWs. Changes to the dispersions, as a result of confinement, orientation and carrier type will affect the velocities. We find that other than the trivial shift of the different valleys in energy with confinement, the velocities are also affected by strong confinement-induced dispersion changes, especially in p-type [110] and [111] NW channels and at less degree in n-type [110] NWs. This causes a large velocity increase with diameter scaling. We further on identify the confining surfaces that are responsible for this. Interestingly, in [110] oriented channels, mostly the (001) confinement is the one which improves the electron velocity, whereas the (1-10) confinement the one that improves the hole velocity.

\section{APPROACH}

The NWs' bandstructure is calculated using the 20 orbital atomistic tight-binding spin-orbit-coupled model $\left(\mathrm{sp}^{3} \mathrm{~d}^{5} \mathrm{~s}^{*}-\mathrm{SO}\right)$ $[8,9,10]$. In this model each atom in the NW is described by 20 orbitals, including spin-orbit-coupling.

The NW description is built on the actual diamond lattice, and each atom is properly accounted in the calculation. It accurately captures the electronic structure and the respective carrier velocities, and inherently includes the effects of quantization and different orientations. The $\mathrm{sp}^{3} \mathrm{~d}^{5} \mathrm{~s}^{*}-\mathrm{SO}$ model was extensively used in the calculation of the electronic properties of nanostructures with excellent agreement to experimental observations on various occasions $[12,13,14]$. It is a compromise between computationally expensive fully abinitio methods, and numerically inexpensive but less accurate effective mass models. In such way, structures of several thousands of atoms can be analyzed [9]. Our calculations involve from 155 to 5500 atoms. We consider infinitely long, cylindrical silicon NWs with hydrogen passivated surfaces [15]. The electronic structure of ultra scaled devices is sensitive to the diameter and orientation $[10,16,17]$. Differences in the shapes of the dispersions between wires of different orientations and diameters, in the number of subbands, as well as the relative differences in their placement in energy, can result is different electronic properties. We consider three different transport orientations [100], [110], and [111] and diameters from $D=3 \mathrm{~nm}$ to $D=12 \mathrm{~nm}$. In this study no relaxation for the NW surfaces is assumed. 


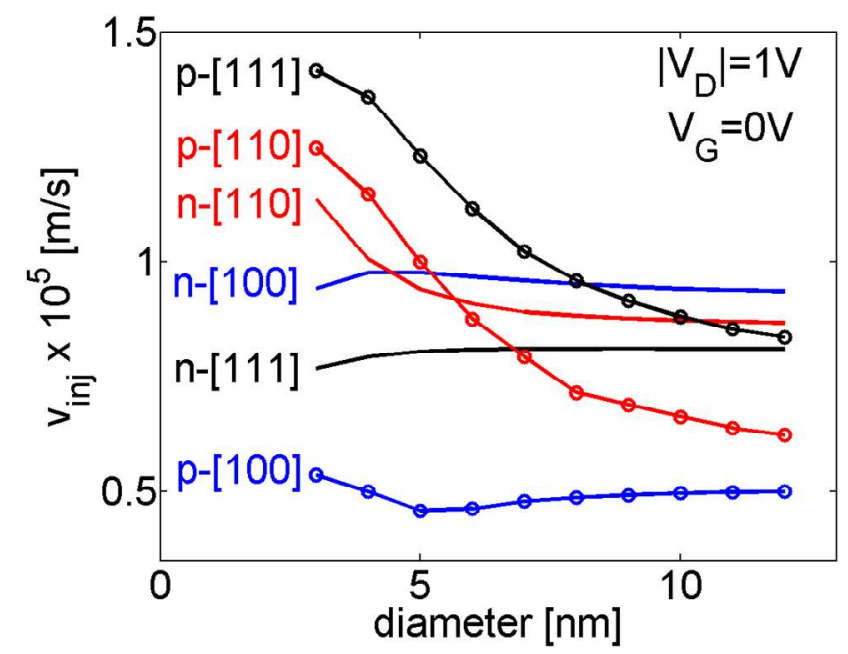

Figure 1. The carrier velocities of cylindrical n-type and p-type NWs in [100], [110], and [111] transport orientations vs. diameter. Non-degenerate conditions and constant potential in the cross section are assumed.

\section{RESULTS AND DISCUSSION}

Figure 1 shows the bandstructure velocity of n-type and ptype cylindrical NWs in the [100], [110] and [111] transport orientations under non-degenerate conditions versus diameter. Large velocity variations with orientation and diameter are observed for some NW categories, while for others the velocities only undergo minor changes. Specifically, for p-type [110] and [111] NWs, the velocity can increase by $\sim 2 \mathrm{X}$ as the diameter decreases. The carrier velocities for the n-type [110] NWs also increase as the diameter scales, however, at a smaller degree of $\sim 50 \%$. The velocities of n-type [100] and [111], as well as the p-type [100] NWs vary only slightly with diameter.

The velocity behavior originates from the NWs dispersions, and how these change under diameter scaling. Examples of the different electronic structures for some NW categories in different orientations and diameters are shown in Fig. 2. Factors that cause velocity variations with diameter are: i) The trivial different energy shift of the various valleys with confinement. This is shown in Fig. $2 \mathrm{a}$ and $2 \mathrm{~b}$ for the [100] ntype NWs of diameters $D=12 \mathrm{~nm}$ and $D=3 \mathrm{~nm}$ respectively, and ii) The change in the subbands' curvature and respectively the effective mass with quantization [10,17]. Figures $2 \mathrm{c}$ and $2 \mathrm{~d}$ show the electronic structure of p-type [110] NWs for diameters $D=12 \mathrm{~nm}$ and $D=3 \mathrm{~nm}$ respectively. The small curvature subbands (large effective mass) of the larger diameter NW become lighter when the diameter is scaled. This effect increases the hole velocities. A very similar behavior is also observed in the case of p-type [111] NWs.

The [100] and [110] orientations are the best for n-type NWs, with the [100] NW performing slightly better at larger diameters, whereas the [110] NW performing slightly better at lower diameters. For p-type NWs, the [111] oriented ones perform better, followed by the [110] ones, whereas the [100]

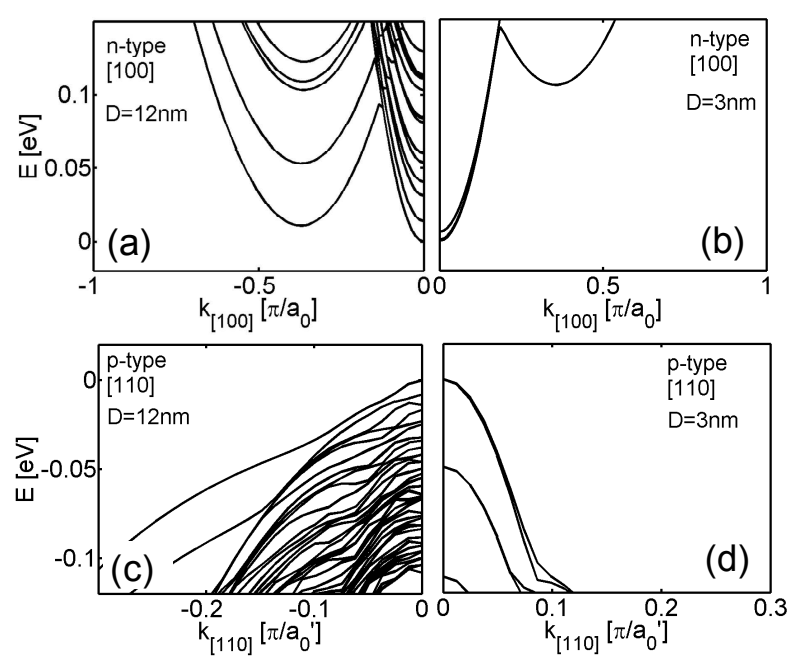

Figure 2. Dispersions of cylindrical NWs. (a) n-type [100] $D=12 \mathrm{~nm}$. (b) ntype [100] $D=3 \mathrm{~nm}$. (c) p-type [110] $D=12 \mathrm{~nm}$. (d) p-type [110] $D=3 \mathrm{~nm}$.

NWs have the lowest carrier velocities from all NW families examined, either n- or p-type. We note that under high concentration a similar trend, but higher velocities have been reported [17].

To explain the mechanism described above, Fig. 3 shows the heavy-hole (100) and (110) energy surfaces for bulk Si. Under quantization, the relevant subbands are picked from different parts of the Brillouin zone (drown lines/arrows) [10, 18]. This results in the variation of the subband curvatures with diameter as previously shown in Fig. $2 \mathrm{c}$ and $2 \mathrm{~d}$ for the cases of $D=12 \mathrm{~nm}$ and $D=3 \mathrm{~nm}$ [110] p-type NWs. Such an effect, but weaker, is also observed for the projected $\Gamma$ valleys of the ntype [110] NW at $D=3 \mathrm{~nm}[10]$. This together with the upward shift in energy of the off- $\Gamma$ valleys improves the electron velocities of this NW with diameter scaling. On the other hand, in the case of n-type [100] NWs, the $\Gamma$ valley bands become heavier with diameter scaling. This tends to decrease the carrier velocities, whereas the upward shift in the off- $\Gamma$ valleys tends to increase them. The final result is that the carrier velocity of n-type [100] NWs is almost invariant of diameter.
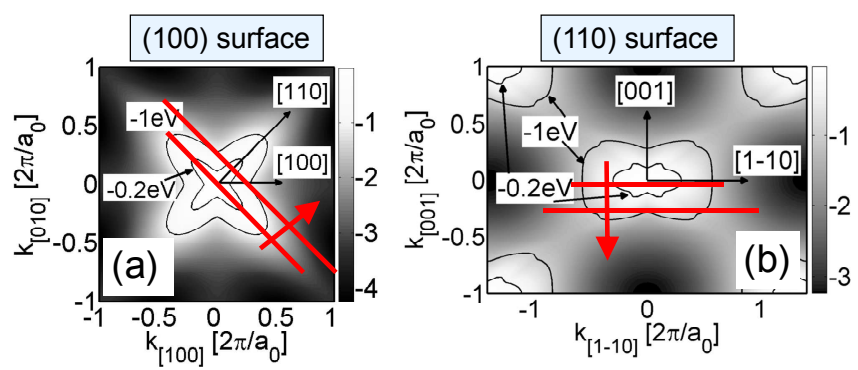

Figure 3. Bulk Si energy surfaces for holes. (a) (100) energy surface. (b) (110) energy surface. The arrows and dashed lines indicate the relevant bands under structure cross section scaling. 


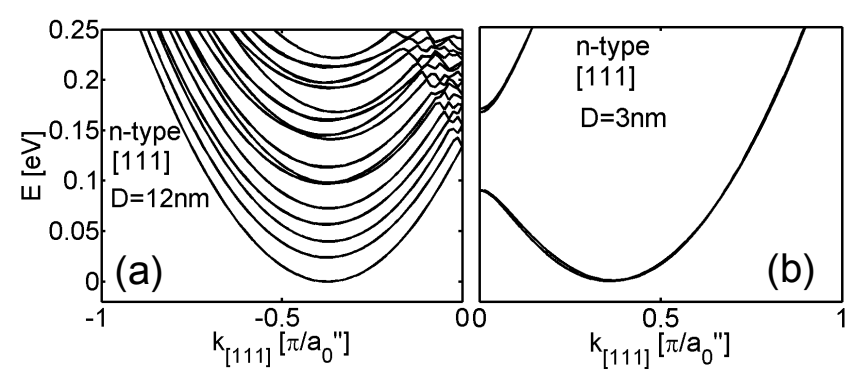

Figure 4. Dispersions of cylindrical NWs. (a) n-type [111] $D=12 \mathrm{~nm}$. (b) n-type [111] $D=3 \mathrm{~nm}$.

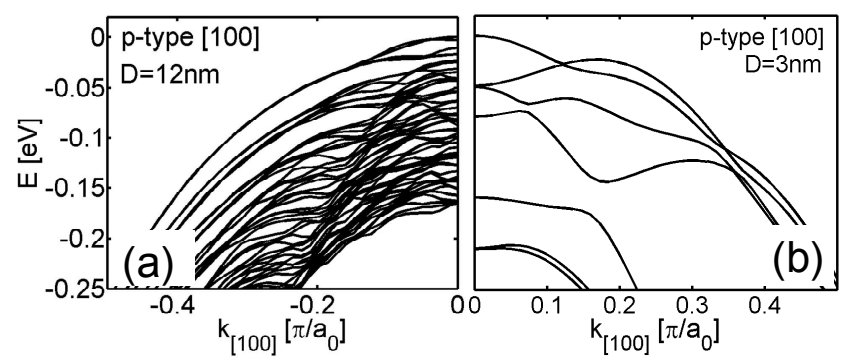

Figure 5. Dispersions of cylindrical NWs. (a) p-type [100] $D=12 \mathrm{~nm}$. (b) p-type $[100] D=3 \mathrm{~nm}$.

The electron velocity in n-type [111] NWs is also independent of diameter scaling. The dispersions for the $D=12 \mathrm{~nm}$ and $D=3 \mathrm{~nm}$ NWs are shown in Fig. $4 \mathrm{a}$ and $4 \mathrm{~b}$, respectively. In this case the dispersion consists of two pairs of 3 -fold degenerate valleys. When the diameter reduces, only a few subbands remain in the dispersion, and their effective mass slightly increases $[10,18]$. This only causes a slight reduction in the electron velocities.

For the p-type [100] NWs, the dispersions for $D=12 \mathrm{~nm}$ and $D=3 \mathrm{~nm}$ are shown in Fig. 5a and $5 \mathrm{~b}$, respectively. The curvature of the dispersions does not change significantly with diameter, and as a result the hole velocities also do not vary. The velocities of this NW remain low, and are lower than the rest NW categories. The reasons are evident from the dispersions as well. The strong oscillating pattern in the curvature of the subbands, and the several flat slope regions keep the velocities low since the velocities are proportional to the slope of the energy dispersions.

As we show further on, in some cases, the different confining surfaces can have a different effect on the velocities. This can be important for the performance assessment of rectangular NWs, or even for 2D ultra-thin-body channels. To demonstrate this, in Fig. 6 we show the dispersions of rectangular [110] NWs with different aspect ratios. NWs in this particular transport orientation have (001) confinement in the height $(H)$ direction, and (1-10) surface confinement in the width $(W)$ direction. Two different geometries are examined, thin and tall NWs, and wide and short ones, as shown by the rectangles in the sub-figures. In the first case, strong (1-10) confinement in the width direction is applied, whereas in the
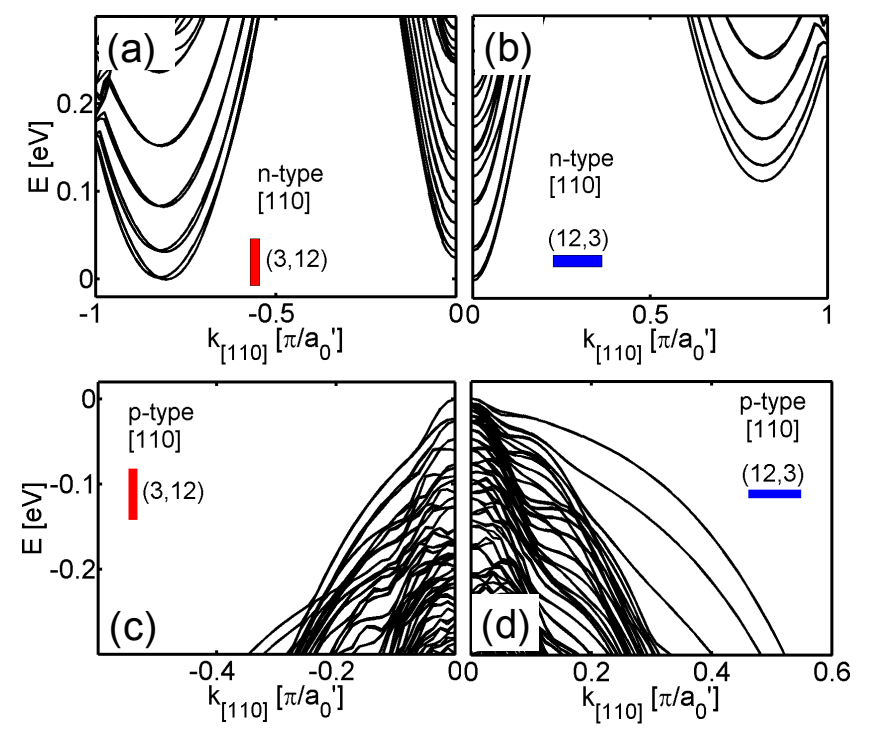

Figure 6. Dispersions of rectangular [110] NWs with different cross section aspect ratios. (a) n-type, $W=3 \mathrm{~nm}, H=12 \mathrm{~nm}$. (b) n-type, $W=12 \mathrm{~nm}, H=3 \mathrm{~nm}$. (c) p-type, $W=3 \mathrm{~nm}, H=12 \mathrm{~nm}$. (d) p-type, $W=12 \mathrm{~nm}, H=3 \mathrm{~nm}$.

latter case, strong (001) confinement in the height direction is applied.

Figure 6a shows the dispersion of the n-type [110] NW with $W=3 \mathrm{~nm}$ and $H=12 \mathrm{~nm}$. Strong (1-10), but weak (001) confinement raises the light mass $\Gamma$ valleys above the heavier mass off- $\Gamma$ valleys. On the other hand, strong (001) but weak (1-10) confinement, raises the heavier mass off- $\Gamma$ valleys above the light mass $\Gamma$ valleys. In the first case, the electron velocity is expected to remain low, whereas in the latter case to be higher. This is exactly the case, as shown in Fig. 7, which shows the carrier velocity of rectangular [110] NWs with different aspect ratios. In this figure, the side which is strongly scaled (at $3 \mathrm{~nm}$ side size) remains constant, whereas the other side varies from $12 \mathrm{~nm}$ down to $3 \mathrm{~nm}$. The data in the far right side of the figure, therefore, indicate the velocities corresponding to the dispersions of Fig. 6. Indeed, the carrier velocities for the conduction band between the wide and short (higher) and the thin and tall (lower) differ in magnitude. As the long side of the two NWs is reduced to $3 \mathrm{~nm}$, forming a channel with $W=H=3 \mathrm{~nm}$, the velocities of both channels increase to the same point. Further confinement of the (1-10) surface (width reduction), however, does not provide any advantage in the velocities. Most of the velocity benefit in ntype [110] channels originates from the (001) confinement.

The quantization behavior of the p-type [110] NWs, on the other hand is reversed. The strongly (1-10) confined, thin and tall, $W=3 \mathrm{~nm}, H=12 \mathrm{~nm}$ NW has subbands with larger curvature than the wide and short, $W=12 \mathrm{~nm}, H=3 \mathrm{~nm} \mathrm{NW}$ as shown in Fig. 3a. The (1-10) confinement provides dispersions with large curvatures and high carrier velocities than the (001) confinement. The carrier velocities of these rectangular NWs are also shown in Fig. 7. Indeed the NW with the strongly confined (1-10) side provides $\sim 40 \%$ higher velocities than the 


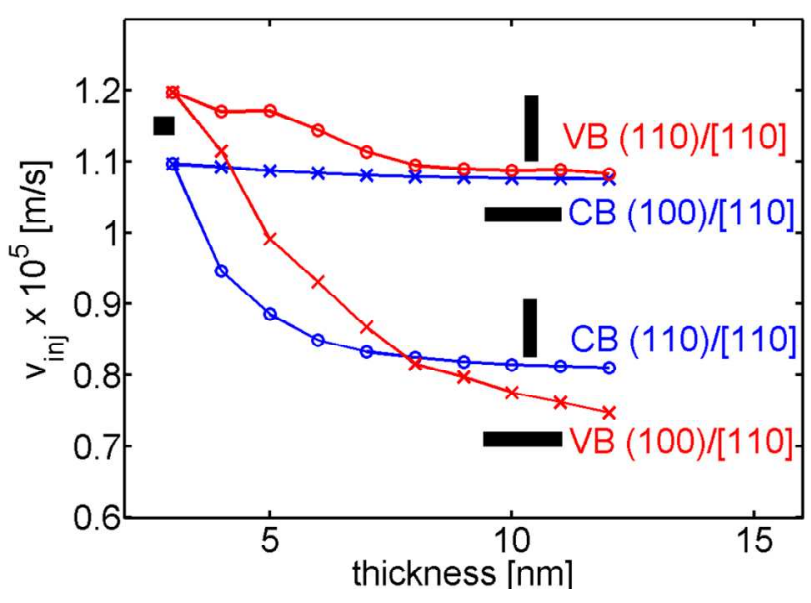

Figure 7. Carrier velocities of rectangular n-type (CB) and p-type (VB) NWs in the [110] transport orientation. One side is kept at $L_{I}=3 \mathrm{~nm}$ (denoted surface), whereas the other varies from $L_{2}=12 \mathrm{~nm}$ down to $L_{2}=3 \mathrm{~nm}$ (filled rectangles). The far left points denote the $L_{1}=L_{2}=3 \mathrm{~nm}$ square NW.

NW with stronger (001) surface confinement (right side of Fig. 7 for the valence band - VB NWs). Scaling the longer side of the NWs to $3 \mathrm{~nm}$ (i.e. moving to the left of Fig. 7) increases the velocities to $1.2 \times 10^{5} \mathrm{~m} / \mathrm{s}$, slightly higher than that of the ntype NWs. From here, the (1-10) side scaling is the one that provides most of the advantage in the hole velocities, whereas (001) surface confinement only offers small improvements. For the [110] transport NWs, therefore, n-type channels are benefited from (001) confinement, whereas p-type ones from (1-10) confinement. Careful size optimization should therefore be applied for CMOS designs that require high velocities for both carrier types. Finally, we mention that for p-type [111] NWs, confinement in any direction provides an increase in the hole velocities. We believe these results can provide design guidance towards high performance NW devices.

\section{CONCLUSION}

A comprehensive analysis of the carrier velocities of $\mathrm{Si}$ NWs is presented, considering: i) n-type and p-type NWs, ii) various orientations and surface quantizations, iii) diameters (cylindrical) and side lengths (rectangular) up to $12 \mathrm{~nm}$. Large velocity variations with orientation and diameter are observed in p-type [110] and [111] NWs, as well as n-type [110] NWs. The velocities of n-type [100] and [111] and p-type [100] NWs do not vary significantly with diameter scaling. Regarding the [110] NWs, the velocity improvements in the n-type case originate from (001) surface confinement, whereas for the ptype case from the (1-10) confinement. The results point towards optimization strategies in the design of NW devices.

\section{ACKNOWLEDGMENT}

This work was supported by the Austrian Climate and Energy Fund, contract 825467. Computational resources of nanoHUB.org are acknowledged. The findings of this work can be verified by the Bandstructure Lab on nanoHUB.org.

\section{REFERENCES}

[1] 1ITRS Public Home Page, http://www.itrs.net/reports.html.

[2] J N. Singh, F. Y. Lim, W. W. Fang, S. C. Rustagi, L. K. Bera, A. Agarwal,C. H. Tung,K. M. Hoe, S. R. Omampuliyur, D. Tripathi, A. O. Adeyeye, G. Q. Lo, N. Balasubramanian, and D. L. Kwong, "Ultranarrow silicon nanowire gate-all-around CMOS devices: Impact of diameter, channel-orientation and low temperature on device performance," Int. Elec. Dev. Meeting, IEDM 2006.

[3] K. H. Cho, K.H. Yeo, Y.Y Yeoh, S. D. Suk, M. Li, J. M. Lee, M.-S. Kim, D.-W. Kim, D. Park, B. H. Hong, Y. C. Jung, and S. W. Hwang, "Experimental evidence of ballistic transport in cylindrical gate-allaround twin silicon nanowire metal-oxide-semiconductor field-effect transistors," Appl. Phys. Lett., 92, 052102, 2008.

[4] A. I. Boukai, Y. Bunimovich, J. T.-Kheli, J.-K. Yu, W. A. G. III, and J. R. Heath, "Silicon nanowires as effective thermoelectric materials," Nature, vol. 451, pp. 168-171, 2008.

[5] A. I. Hochbaum, R. Chen, R. D. Delgado, W. Liang, E. C. Garnett, M. Najarian, A. Majumdar, and P. Yang, "Enhanced thermoelectric performance of rough silicon nanowires," Nature, vol. 451, pp. 163-168, 2008.

[6] C. B. Winkelmann, I. Ionica, X. Chevalier, G. Royal, C. Bucher, and V. Bouchiat, "Optical switching of porphyrin-coated silicon nanowire field effect transistors," Nano Lett., 7, 6, pp. 1454-1458, 2007.

[7] S. Huang, and Y. Chen, "Ultrasensitive fluorescence detection of single protein molecules manipulated electrically on Au nanowire," Nano Lett., vol. 8, no. 9, pp. 2829-2833, 2008.

[8] T. B. Boykin, G. Klimeck, and F. Oyafuso, "Valence band effectivemass expressions in the $\mathrm{sp} 3 \mathrm{~d} 5 \mathrm{~s} *$ empirical tight-binding model applied to a Si and Ge parametrization," Phys. Rev. B, vol. 69, pp. 115201115210,2004

[9] G. Klimeck, S. Ahmed, H. Bae, N. Kharche, S. Clark, B. Haley, S. Lee, M. Naumov, H. Ryu, F. Saied, M. Prada, M. Korkusinski, and T. B. Boykin, "Atomistic simulation of realistically sized nanodevices using NEMO 3-D - Part I: Models and benchmarks," IEEE Trans. Electr. Dev., vol. 54, no. 9, pp. 2079-2089, 2007.

[10] N. Neophytou, A. Paul, M. Lundstrom, and G. Klimeck, "Bandstructure effects in silicon nanowire electron transport," IEEE Trans. Elect. Dev., vol. 55, no. 6, pp. 1286-1297, 2008.

[11] A. Rahman, J. Guo, S. Datta, and M. Lundstrom, "Theory of ballistic nanotransistors," IEEE Trans. Electr. Dev., vol. 50, no. 9, pp. 18531864, 2003.

[12] J. Wang, PhD thesis, Purdue University, 2005.

[13] N. Kharche, M. Prada, T. B. Boykin, and G. Klimeck, "Valley splitting in strained silicon quantum wells modeled with $2^{\circ}$ miscuts, step disorder, and alloy disorder," Appl. Phys. Lett., vol. 90, no. 9, p. 092109, 2007.

[14] R. Rahman, C. J. Wellard, F. R. Bradbury, M. Prada, J. H. Cole, G. Klimeck, and L. C. L. Hollenberg, "High precision quantum control of single donor spins in silicon,” Phys. Rev. Lett., vol 99, no. 3, p. 036403 , 2007.

[15] S. Lee, F. Oyafuso, P. Von, Allmen, and G. Klimeck, "Boundary conditions for the electronic structure of finite-extent embetted semiconductor nanostructures," Phys. Rev. B, vol. 69, pp. 045316045323,2004

[16] N. Neophytou, A. Paul, and G. Klimeck, "Bandstructure effects in silicon nanowire hole transport," IEEE Trans. Nanotechnol., vol. 7, no. 6, pp. 710-719, 2008.

[17] N. Neophytou, S. G. Kim, G. Klimeck, and H. Kosina, "On the Bandstructure Velocity and Ballistic Current of Ultra Narrow Silicon Nanowire Transistors as a Function of Cross Section Size, Orientation and Bias," J. Appl. Phys, vol. 107, no. 113701, 2010.

[18] N. Neophytou and G. Klimeck, "Design space for low sensitivity to size variations in [110] PMOS nanowire devices: The implications of anisotropy in the quantization mass," Nano Lett.,vol. 9, no. 2, pp. 623$630,2009$. 